\title{
Neighborhood Characteristics and Hypertension
}

\author{
Mahasin S. Mujahid, ${ }^{\mathrm{a}}$ Ana V. Diez Roux, ${ }^{\mathrm{a}}$ Jeffrey D. Morenoff, ${ }^{\mathrm{b}}$ Trivellore E. Raghunathan, ${ }^{\mathrm{c}}$ \\ Richard S. Cooper, ${ }^{\mathrm{d}}$ Hanyu Ni, ${ }^{\mathrm{e}}$ and Steven Shea ${ }^{\mathrm{f}, \mathrm{g}}$
}

Background: The goal of this study was to investigate crosssectional associations between features of neighborhoods and hypertension and to examine the sensitivity of results to various methods of estimating neighborhood conditions.

Methods: We used data from the Multi-Ethnic Study of Atherosclerosis on 2612 individuals 45-85 years of age. Hypertension was defined as systolic blood pressure above $140 \mathrm{~mm} \mathrm{Hg}$, diastolic pressure above $90 \mathrm{~mm} \mathrm{Hg}$, or use of antihypertensive medications. Neighborhood (census tract) conditions potentially related to hypertension (walking environment, availability of healthy foods, safety, social cohesion) were measured using information from a separate phone survey conducted in the study neighborhoods. For each neighborhood we estimated scale scores by aggregating residents' responses using simple aggregation (crude means) and empirical Bayes estimation (unconditional, conditional, and spatial). These estimates of neighborhood conditions were linked to each study participant based on the census tract of residence. Two-level binomial regression methods were used to estimate adjusted associations between neighborhood conditions and hypertension.

Results: Residents of neighborhoods with better walkability, availability of healthy foods, greater safety, and more social cohesion were less likely to be hypertensive (relative prevalence [95\% confidence interval] for 90 th vs. 10th percentile of conditional empirical Bayes estimate $=0.75[0.64-0.88], 0.72$ [0.61-0.85], 0.74 [0.63$0.86]$, and 0.69 [0.57-0.83]), respectively, after adjusting for site, age, sex, income, and education. Associations were attenuated and often disappeared after additional adjustments for race/ethnicity.

Submitted 30 May 2007; accepted 31 December 2007; posted 12 May 2008. From the a Department of Epidemiology, University of Michigan School of

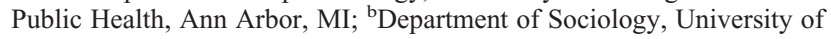
Michigan College of Literature, Science, and the Arts, Ann Arbor, MI; ${ }^{\mathrm{c}}$ Department of Biostatistics, University of Michigan School of Public Health, Ann Arbor, MI; ${ }^{\mathrm{d} D e p a r t m e n t}$ of Preventive Medicine, Loyola University, Chicago, IL; ' ${ }^{\mathrm{D}}$ ivision of Epidemiology and Clinical Applications, National Heart, Lung, and Blood Institute, National Institutes of Health, Bethesda, MD; and Departments of ${ }^{\mathrm{f}}$ Medicine and ${ }^{\mathrm{g}}$ Epidemiology, Columbia University, New York, NY.

Supported by R01 HL071759. Multi-Ethnic Study of Atherosclerosis is supported by contracts N01-HC-95159 through N01-HC-95165, N01HC-95169, and R01 HL071759 from the National Heart, Lung, and Blood Institute.

Correspondence: Ana V. Diez Roux, Center for Social Epidemiology and Population Health, University of Michigan School of Public Health, 109 Observatory Street Rm 3671, Ann Arbor, MI 48104. E-mail: adiezrou@umich.edu.

Copyright (C) 2008 by Lippincott Williams \& Wilkins

ISSN: $1044-3983 / 08 / 1904-0590$

DOI: 10.1097/EDE.0b013e3181772cb2
Conclusion: Neighborhood walkability, food availability, safety, and social cohesion may be mechanisms that link neighborhoods to hypertension.

(Epidemiology 2008;19: 590-598)

iving in socioeconomically disadvantaged neighborhoods -is associated with greater cardiovascular disease (CVD) prevalence, incidence, and mortality. ${ }^{1-6}$ These associations may be partly attributable to the effects of neighborhood characteristics on CVD risk factors. ${ }^{7}$ Evidence suggests that neighborhood conditions are related to body mass index (BMI), smoking, and poor diets. ${ }^{7-11}$ Several studies have examined the association of neighborhoods with blood pressure outcomes ${ }^{3,6,12-14}$ with some documenting associations with neighborhood disadvantage $\mathrm{e}^{3,12-14}$ and others failing to document any effect. ${ }^{6}$

A major limitation of past work on neighborhood environments and CVD has been the use of neighborhood socioeconomic position as the neighborhood-level variable investigated. The use of this measure raises methodologic questions regarding the extent to which aggregate and individual-level socioeconomic position can be meaningfully separated empirically. ${ }^{15}$ It may also result in incorrect estimates of neighborhood health effects if neighborhood socioeconomic position is a poor proxy for the true neighborhood construct of interest. The use of area socioeconomic position does not allow investigation of the specific causal processes linking neighborhood environments with CVD risk. ${ }^{7,16}$ Consequently, researchers have called for work that examines how specific neighborhood features are related to health outcomes based on conceptual models of the processes involved. ${ }^{7,11,16-18}$

Blood pressure may be related to neighborhoods through multiple mechanisms. Potential hypertension-inducing features of neighborhoods include limited access to resources conducive to healthy lifestyles (availability of healthy foods, walking environment) and an excess of neighborhood stressors (violence and poor social cohesion). Although it is sometimes possible to measure features of neighborhoods using existing databases,,${ }^{19,20}$ these sources are often limited. Studies have begun to explore the use of innovative techniques for directly collecting data on neighborhood environments ${ }^{21-23}$ including systematic social observation $^{21,22,24}$ and surveys of area residents. ${ }^{23,25,26}$ 
We used data from the Multi-Ethnic Study of Atherosclerosis to examine cross-sectional associations of specific features of residential environments with blood pressure. Our analysis had 3 aims: (1) to develop measures of neighborhood environments using traditional and novel methods of estimation, (2) to estimate cross-sectional associations between specific features of neighborhood environments and hypertension, and (3) to test the sensitivity of these associations to various methods of scale estimation. Based on a conceptual framework, ${ }^{7}$ we hypothesized that residents living in neighborhoods with poor walking environments, limited access to healthy foods, lack of safety, and lack of cohesiveness among neighbors would have a higher prevalence of hypertension.

\section{METHODS}

The study sample is comprised of participants of the Multi-Ethnic Study of Atherosclerosis, which is a longitudinal study of 6814 adults aged 45-84 years without clinical cardiovascular disease at baseline. ${ }^{27}$ Participants were recruited between August 2000 and July 2002 from 6 study sites (Baltimore, MD; Chicago, IL; Forsyth County, NC; Los Angeles, CA; New York, NY; and St. Paul, MN). Each study site recruited participants from locally available sources (lists of residents, lists of dwellings, telephone exchanges). Participation rates among those screened and deemed eligible was $60 \%$. These analyses are based on baseline data for the 3 sites (Baltimore, MD; Forsyth County, NC; and New York City, NY) for which additional neighborhood-level data were collected.

Hypertension was defined as systolic blood pressure above $140 \mathrm{~mm} \mathrm{Hg}$, diastolic pressure above $90 \mathrm{~mm} \mathrm{Hg}$, or use of antihypertensive medications. ${ }^{28}$ Resting seated blood pressure was measured 3 times using an automated oscillometric device. The average of the last 2 measurements was used for analysis. ${ }^{29}$ Medication use was assessed through questionnaire. Results were similar when we used continuous blood pressure adjusted for medication.

Information on neighborhoods was obtained through an ancillary study designed to measure neighborhood characteristics at 3 sites. A sample of 5988 individuals residing in the same neighborhoods (census tracts) as study participants were asked to rate their neighborhood via a telephone survey. Surveying people who were not study participants avoids the potential for same-source bias (eg, individuals who are sedentary may rate their neighborhoods worse on recreational resources than their more active counterparts, irrespective of the actual conditions of the neighborhood) and also allows aggregation across multiple respondents yielding a more valid measure. The survey recruited a median of 8 individuals per neighborhood (census tract) (range 1-62) and a total of 576 neighborhoods were represented (276 in Maryland, 71 in North Carolina, and 297 in New York). Additional details on the survey are described elsewhere. ${ }^{23}$

The telephone questionnaire asked individuals to report on conditions for the area about a mile around their home. We
TABLE 1. Neighborhood Scale Items Included in the Phone Survey to Assess Neighborhood Conditions, the Multi-Ethnic Study of Atherosclerosis Neighborhood Study

\begin{tabular}{|c|c|}
\hline Neighborhood Dimension & Scale Item \\
\hline Walking environment & $\begin{array}{l}\text { My neighborhood offers many opportunities to } \\
\text { be physically active } \\
\text { Local sports clubs and other facilities in my } \\
\text { neighborhood offer many opportunities to } \\
\text { get exercise } \\
\text { It is pleasant to walk in my neighborhood } \\
\text { The trees in my neighborhood provide enough } \\
\text { shade } \\
\text { In my neighborhood it is easy to walk places } \\
\text { I often see other people walking in my } \\
\text { neighborhood } \\
\text { I often see other people exercise (for example, } \\
\text { jog, bicycle, play sports) in my } \\
\text { neighborhood }\end{array}$ \\
\hline $\begin{array}{l}\text { Availability of healthy } \\
\text { foods }\end{array}$ & $\begin{array}{l}\text { A large selection of fresh fruits and vegetables } \\
\text { is available in my neighborhood } \\
\text { The fresh fruits and vegetables in my } \\
\text { neighborhood are of high quality } \\
\text { A large selection of low fat products is } \\
\text { available in my neighborhood }\end{array}$ \\
\hline Safety & $\begin{array}{l}\text { I feel safe walking in my neighborhood day or } \\
\text { night } \\
\text { Violence is not a problem in my neighborhood } \\
\text { My neighborhood is safe from crime }\end{array}$ \\
\hline Social cohesion & $\begin{array}{l}\text { People around here are willing to help their } \\
\text { neighbors } \\
\text { People in my neighborhood generally get } \\
\text { along with each other } \\
\text { People in my neighborhood can be trusted } \\
\text { People in my neighborhood share the same } \\
\text { values }\end{array}$ \\
\hline
\end{tabular}

assessed 4 neighborhood dimensions: walking environment (7 items), availability of healthy foods ( 3 items), safety (3 items), and social cohesion (4 items) (Table 1). Scale items representing each of the dimensions were adapted from published work whenever possible. ${ }^{26,30-34}$ Respondents indicated agreement with items on a 5-point Likert scale $(1=$ strongly agree to $5=$ strongly disagree) (Table 1 ). Items within each neighborhood dimension had good internal consistency (Cronbach's $\alpha$ : 0.73-0.78) and test-retest reliabilities (intraclass correlations $=0.60-0.88) .{ }^{23}$ Neighborhood scores were calculated using various methods to aggregate responses across individuals within each neighborhood (described below) and then linked to participants in the Multi-Ethnic study of Atherosclerosis using the baseline residential addresses.

\section{Computation of Neighborhood Estimates}

We contrasted 4 methods of aggregating individual survey responses within neighborhoods to estimate the measure for the neighborhood as a whole: crude means and 3 different versions of empirical Bayes estimates (uncondi- 
tional, conditional, and spatial) as described below. Census tracts were used as proxies for neighborhoods based on prior work, indicating that there is better agreement in survey responses across individuals within census tracts than within larger units. ${ }^{23}$ These 4 methods are as follows:

1. Crude means: $\bar{Y}_{00 k}=\frac{1}{n_{j k} n_{i}} \sum_{j=1}^{n_{j k}} \sum_{i=1}^{n_{i}} x_{i j k}$

where $\bar{Y}_{00 \mathrm{k}}$ is the crude mean for neighborhood $k$ for a particular neighborhood dimension, $x_{i j k}$ is the response to the $i$ th item for the $j$ th person in the $k$ th neighborhood, $n_{j k}$ is the number of individuals $j$ within neighborhood $k$, and $n_{i}$ is the number of scale items.

Using this approach, we calculated a neighborhood mean scale score for each neighborhood $k$ by summing across $i$ items within an individual, summing across $j$ individuals within neighborhood $k$ and dividing by the number of items $i$ and the number of individuals $j$ in neighborhood $k$. The strength of using the crude mean is its simplicity; there are few assumptions involved. However, because our withinneighborhood sample size differs across neighborhoods (range 1-62 respondents per neighborhood), crude mean estimates could introduce measurement error in neighborhoods where only a few respondents were queried.

2. Unconditional empirical Bayes: $\beta_{00 k}^{*}=\hat{\lambda}_{\beta_{k}} \hat{\beta}_{00 k}+$ $\left(1-\hat{\lambda}_{\beta k}\right) \hat{\gamma}_{000}$

where $\beta_{00 k}^{*}$ is the unconditional empirical Bayes estimate of the mean scale score for neighborhood $k$ for a particular neighborhood dimension, $\hat{\lambda}_{\beta k}$ is the estimated reliability of the neighborhood level measure, $\hat{\beta}_{00 k}$ is the model estimated crude mean for neighborhood $k, \hat{\gamma}_{000}$ is the estimated mean scale score pooled across neighborhoods. ${ }^{35}$

Unconditional empirical Bayes estimates were derived from 3-level hierarchical linear models that account for the nested structured of the data (ie, scale items nested within individuals who are nested within neighborhoods). The level 1 model: $Y_{i j k}=b_{o j k}+e_{i j k}$, represents the $i$ th response item for the $j$ th person in the $k$ th neighborhood as a function of the person-specific mean score across all items corresponding to a neighborhood dimension $\left(b_{o j k}\right)$, and an error term $\left(e_{i j k}\right)$ that is normally distributed with a mean 0 and variance $\sigma^{2}$. The level 2 model: $b_{0 j k}=\beta_{0 o k}+\alpha_{0 j k}$ models the person-specific mean score for a person $j$ in neighborhood $k$ as a function of the neighborhood specific mean $\left(\beta_{\text {ook }}\right)$ and a random effect for person $j$ in neighborhood $k\left(\alpha_{0 j k}\right)$ that is normally distributed with mean 0 and variance $\tau_{b}$. The level 3 model: $\beta_{0 o k}=$ $\gamma_{000}+U_{\text {ook }}$, models the neighborhood specific mean score for neighborhood $k$ as a function of the overall mean score $\left(\gamma_{000}\right)$ and a random effect for neighborhood $k\left(U_{00 k}\right)$ that is normally distributed with mean 0 and variance $\tau_{\eta}{ }^{23}$ The empirical Bayes estimate is a weighted average of the crude mean for each neighborhood $k$ and the crude mean across all neighborhoods in the study. The weights are proportional to the reliability of the neighborhood measure. The reliability is estimated by

$$
\operatorname{reliability}\left(\hat{\beta}_{00 k}\right)=\frac{\tau_{\eta}}{\tau_{\eta}+\left\{\sum_{1}^{j}\left[\tau_{b}+\sigma^{2} / n_{j k}\right]^{-1}\right\}^{-1}}
$$

The greater the variance across neighborhoods and the greater the sample size within neighborhoods, the greater the reliability. Hence, a neighborhood measure can be unreliable if (1) there is poor agreement among the individuals within the neighborhood as to the neighborhood conditions, or (2) there is small within-neighborhood sample size. Because reliability ranges from 0 to 1 , with 1 representing a perfectly reliable measure, those neighborhoods that have good reliability have little shrinkage; however, those neighborhoods with poor reliability will have greater shrinkage toward the overall mean. Thus, the strength of this approach is that it borrows information from other neighborhoods to improve the estimate for unreliable neighborhoods. A limitation is that these estimates might introduce excessive shrinkage and mask differences between neighborhoods. On average the neighborhood scales within our study have good reliability with values of $0.73,0.64,0.77$, and 0.68 for neighborhood walking environment, availability of healthy foods, safety, and social cohesion, respectively. ${ }^{23}$ Reliabilities were similar when item responses were modeled using an ordered logit, and there was no evidence of important violations of normality assumptions of the residuals. Hence, the simpler linear models that allow estimations of means were used in all community survey analyses. ${ }^{23}$

3. Conditional empirical Bayes: $\beta_{00 k}^{*}=\hat{\lambda}_{\beta_{k}} \hat{\beta}_{00 k}+$ where $\beta_{00 k}^{*}$ is the conditional empirical Bayes estimate for neighborhood $k$ for a particular neighborhood dimension, $\hat{\lambda}_{\beta k}$ is the estimated reliability of the neighborhood level measure, $\hat{\beta}_{0 o k}$ is model estimated crude mean for neighborhood $k, \hat{\gamma}_{000}$ is the estimated grand neighborhood mean, when the neighborhood-level covariates $W_{k_{1}}$ and $W_{k_{2}}$ are $0, \hat{\gamma}_{001}, \hat{\gamma}_{002}$ are the parameter estimates for neighborhood socioeconomic position factor scores, $W_{k_{1}}, W_{k_{2}}$ are census-derived measure of neighborhood socioeconomic position. ${ }^{35}$

The conditional empirical Bayes estimate is derived similarly to the unconditional empirical Bayes estimate. The difference is reflected in the level 3 model, which includes measures of neighborhood socioeconomic position in the estimation of the neighborhood-specific mean scale score. Consequently, this conditional empirical Bayes estimator pulls less reliable neighborhoods toward the mean of similar neighborhoods instead of to the mean across all neighborhoods in the study population. Similar neighborhoods are defined as those neighborhoods that have comparable socioeconomic profiles using census-derived indicators of neigh- 
borhood socioeconomic position across various dimensions. Information on indicators of socioeconomic position for neighborhoods (census tracts) was obtained from the 2000 US census. Based on factor analyses of 19 variables (representing the dimensions of racial/ethnic composition, family structure, housing, area crowding, residential stability, education, employment, occupation, and income/wealth) we identified 2 factors that accounted for $83 \%$ of the variance (factor 1: \% of vacant housing, \% of houses without a telephone, $\%$ of houses without a vehicle, $\%$ of unemployed individuals, $\%$ of poverty, median household income; factor 2: $\%$ with at least a bachelors degree, $\%$ of nonmanagerial occupation, $\%$ of households with interest, dividends, or net rental income). These 2 factors were included as predictors in the conditional empirical Bayes estimation.

An advantage of this conditional approach is that it uses information on the relationship between census characteristics and the scale scores to help improve the prediction for neighborhoods with few respondents. If the measures of socioeconomic position are good predictors of the neighborhood conditions, then conditional empirical Bayes estimates may provide better estimates than unconditional empirical Bayes estimates. The unconditional and conditional empirical Bayes estimates will be similar if socioeconomic position measures are poor predictors of the scales. A limitation of this approach is that, because neighborhood socioeconomic position is used to predict the specific features, it may not be possible to estimate the effects of these features independent of neighborhood socioeconomic position. We have some indication that our neighborhood scales are mildly to moderately associated with neighborhood socioeconomic position. ${ }^{23}$

\section{Spatial empirical Bayes: $\beta_{00 k}^{*}=w_{k} \bar{Y}_{0 o k}+\left(1-w_{k}\right) \theta$,}

where $\beta_{00 k}^{*}$ is the spatial empirical Bayes estimate for neighborhood $k$ for a particular neighborhood dimension, $\bar{Y}_{\text {ook }}$ is the crude mean for neighborhood $k$ for a particular neighborhood dimension, $w_{k}$ is a weight for each neighborhood $k, \theta$ is the mean of the surrounding neighborhoods. ${ }^{36}$

The shrinkage estimator for the spatial empirical Bayes estimate was developed using GeoDa, a spatial data analysis software package. ${ }^{36-38}$ The spatial empirical Bayes estimate is a weighted average of the crude mean and the mean of the surrounding neighborhoods. Surrounding neighborhoods are defined as those surrounding areas (tracts) that have common borders or vertices with a particular census tract. The weight: $w_{k}=\frac{\phi}{\phi+\left(\varphi / n_{j k}\right)}$ is a function of the interindividual variance in the person-scale scores of the surrounding neighborhoods $(\phi)$ divided by the sum of the variances of the surrounding neighborhoods $(\phi)$ and local neighborhood $\left(\varphi / n_{j k}\right)$. If the variance in the local neighborhood is small (ie, $\varphi$ is small) or $n_{j k}$ is large, the weight will approximately be 1 and the spatial
Bayes estimate will equal the crude mean. However, if the variance in the local neighborhood is large (ie, $\varphi$ is large or $n_{j k}$ is small) then more weight is given to the mean observed in the surrounding neighborhood and thus more shrinkage occurs. The advantage of this approach is that "unreliable" neighborhoods are pulled toward the mean of the surrounding area instead of the larger study area. This method has limited utility if there is very little spatial patterning. Local Moran's I estimates, an indicator of the amount of spatial patterning, ${ }^{36}$ indicated moderate spatial correlation (in Maryland, 0.26, $0.24,0.41$, and 0.12 for walking environment, availability of healthy foods, safety, and social cohesion, respectively; in New York, 0.23, 0.27, 0.42, and 0.11; and in North Carolina, $0.29,0.25,0.61$, and 0.34 ).

Individual-level variables included study site, age, sex, race/ethnicity (white, non-Hispanic; African American, nonHispanic; Hispanic), education $(<$ high school; high school diploma; some college/technical school; college graduate and beyond), income $(<\$ 25,000 ; \$ 25,000-\$ 49,999 ; \$ 50,000-$ $\$ 75,000 ; \$ 75,000+)$, and time lived in neighborhood $(<20$ years, $20+$ years).

Due to the limitations in calculating an intraclass correlation coefficient in the case of binary outcomes ${ }^{39,40}$ the neighborhood clustering of hypertension was investigated using the median odds ratio (OR). ${ }^{40}$ The median OR is the median of all possible ORs obtained by comparing the outcomes of 2 persons chosen at random from different neighborhoods (with the person with the highest probability of the outcome being placed in the numerator so that the OR can never be $<1) .{ }^{40}$ Larger median OR indicate larger betweenneighborhood variation in the outcome.

Two-level binomial regression was used to investigate associations of hypertension with neighborhood characteristics before and after adjustment for individual level covariates. Exponentiated coefficients from these models were used to estimate adjusted prevalence ratios of hypertension comparing the 90th to the 10th percentile of each neighborhood dimension; 95\% confidence intervals (CIs) were also computed. Neighborhood dimensions were investigated in separate models and the 4 types of estimates (crude mean and unconditional, conditional, and spatial empirical Bayes) were contrasted. We also investigated a summary measure of neighborhood conditions created by summing the respective z-scores of the 4 neighborhood dimensions.

We investigated whether associations of neighborhood characteristics with hypertension were heterogeneous across study site, race/ethnicity, and time lived in neighborhood by including two-way interactions in the statistical models. We investigated these interactions for 3 reasons. First, features of sites such as urbanicity, transportation, and land use mix may modify the relationship between neighborhood features and hypertension. Second, neighborhoods may have more of an impact on vulnerable populations for which race/ethnicity is 
a proxy. And third, individuals must be exposed to poor neighborhood conditions over a substantial period of time to adversely impact health. Interactions were retained in models if $P<0.10$.

\section{RESULTS}

Of the 3265 Multi-Ethnic Study of Atherosclerosis participants at the 3 sites, 2963 (91\%) were geocoded; 2612 had complete information on all covariates of interest $(80 \%)$ and were included in analyses (Table 2). The mean age was $62(\mathrm{SD}=10.0), 54 \%$ were women; $43 \%$ were white (nonHispanic), 42\% were African American (non-Hispanic), and $16 \%$ were Hispanic. Fifty percent of the sample was hypertensive. Hypertension prevalence was highest in blacks and lowest in whites. Hypertension prevalence also decreased with increasing income and education.

A total of 495 neighborhoods were represented in the analyses with a median of 3 participants per neighborhood (range 1-50). As expected due to the effects of shrinkage, variability in neighborhood-level measures was smaller for the shrinkage estimates (the 3 empirical Bayes estimates)

TABLE 2. Selected Sample Characteristics and Prevalence of Hypertension by Sociodemographic Characteristics for the 2612 Multi-Ethnic Study of Atherosclerosis Participants Included in the Analyses

\begin{tabular}{|c|c|c|}
\hline & No. $(\%)$ & \% Hypertension \\
\hline \multicolumn{3}{|l|}{ Study site } \\
\hline Baltimore & $870(33)$ & 50 \\
\hline New York & $903(35)$ & 45 \\
\hline North Carolina & $839(32)$ & 52 \\
\hline \multicolumn{3}{|l|}{$\mathrm{Age}^{\mathrm{a}} ; \mathrm{yrs}$} \\
\hline $45-60$ & $735(28)$ & 30 \\
\hline $60-75$ & $743(29)$ & 48 \\
\hline $75+$ & $1134(43)$ & 62 \\
\hline \multicolumn{3}{|l|}{ Sex } \\
\hline Male & $1198(46)$ & 46 \\
\hline Female & $1414(54)$ & 51 \\
\hline \multicolumn{3}{|l|}{ Race } \\
\hline White & $1109(43)$ & 42 \\
\hline Black & $1084(42)$ & 58 \\
\hline Hispanic & $419(16)$ & 44 \\
\hline \multicolumn{3}{|l|}{ Education } \\
\hline$<$ High school diploma & $353(14)$ & 60 \\
\hline High school diploma & $537(21)$ & 54 \\
\hline Some college & 765 (29) & 49 \\
\hline College graduate + & 957 (37) & 42 \\
\hline \multicolumn{3}{|l|}{ Income } \\
\hline$<\$ 24,999$ & $684(26)$ & 60 \\
\hline$\$ 25,000-\$ 49,999$ & $832(32)$ & 51 \\
\hline$\$ 50,000-\$ 74,999$ & $525(20)$ & 45 \\
\hline$\geq \$ 75,000$ & $571(22)$ & 37 \\
\hline
\end{tabular}

${ }^{\mathrm{a}}$ Mean $(\mathrm{SD})=62.3(10.0)$. than for the crude means. For example, the mean (SD) for availability of healthy foods was $3.30(0.61)$ for crude means and 3.22 (0.37) for unconditional empirical Bayes. Correlations between crude neighborhood means for the measures ranged from 0.38 for availability of healthy foods and social cohesion to 0.70 for safety and social cohesion.

Higher income and education were associated with greater neighborhood walkability, availability of healthy foods, safety, and social cohesion (Table 3). Mean neighborhood conditions were poorer for minorities than for whites, with Hispanics having the lowest mean value across all neighborhood dimensions. Study participants in New York lived in neighborhoods with better average walking environments and availability of healthy foods, but with worse safety and social cohesion.

The median OR for between-neighborhood variability in hypertension was 1.47 (between-neighborhood variance = 0.1582 ) and was reduced to 1.21 (between-neighborhood variance $=0.03872$ ) when age, sex, education, income, and race/ethnicity were added to the model. Residents of neighborhoods with better walking environments, more availability of healthy foods, greater safety, and greater social cohesion were less likely to be hypertensive after adjusting for site, age, and sex (relative prevalence for the 90th versus 10th percentile for crude means $[95 \% \mathrm{CI}]=0.80[0.72-0.89]$, 0.83 [0.75-0.93], 0.77 [0.68-0.87], and 0.83 [0.74-0.93], respectively) (Table 4). These associations were attenuated but persisted after additional adjustments for education and income (except in the case of spatial Bayes estimates for social cohesion, for which associations disappeared after adjustment). Higher values of the combined neighborhood measure were also associated with lower prevalence of hypertension after adjustment for site, age, sex, education, and income (relative prevalence for the 90th vs. 10th percentile for crude means $=0.86[95 \% \mathrm{CI}=0.77-0.96]$ ). All associations were attenuated after additional adjustment for race/ethnicity.

In general, associations were slightly stronger for the empirical Bayes estimation methods (Table 4). For availability of healthy foods, the relative prevalence of hypertension was 0.89 for crude means but $0.80,0.72$, and 0.65 for unconditional, conditional, and spatial empirical Bayes respectively, after adjusting for site, age, sex, education, and income. There was no statistical evidence of interactions between neighborhood dimensions and time lived in neighborhood, study site, or race/ethnicity $(P>0.10$ for all 10 tests for heterogeneity).

\section{DISCUSSION}

Individuals residing in neighborhoods with better walkability, availability of healthy foods, safety, and social cohesion had a lower probability of being hypertensive than their counterparts in worse neighborhoods. These associations per- 
TABLE 3. Mean Neighborhood Characteristics by Sociodemographic Characteristics of Participants, the Multi-Ethnic Study of Atherosclerosis Neighborhood Study

\begin{tabular}{|c|c|c|c|c|}
\hline & $\begin{array}{c}\text { Walking } \\
\text { Environment }\end{array}$ & $\begin{array}{l}\text { Healthy } \\
\text { Foods }\end{array}$ & Safety & $\begin{array}{c}\text { Social } \\
\text { Cohesion }\end{array}$ \\
\hline Overall mean (SD) & $3.59(0.43)$ & $3.30(0.61)$ & $3.19(0.67)$ & $3.36(0.51)$ \\
\hline \multicolumn{5}{|l|}{ Study site } \\
\hline Baltimore & 3.59 & 3.35 & 3.28 & 3.61 \\
\hline New York & 3.69 & 3.43 & 3.11 & 3.24 \\
\hline North Carolina & 3.67 & 3.23 & 3.88 & 3.84 \\
\hline$P^{\mathrm{a}}$ & $<0.001$ & $<0.001$ & $<0.001$ & $<0.001$ \\
\hline \multicolumn{5}{|l|}{ Age } \\
\hline 45-60 & 3.62 & 3.32 & 3.37 & 3.51 \\
\hline $60-75$ & 3.64 & 3.33 & 3.44 & 3.56 \\
\hline $75+$ & 3.67 & 3.36 & 3.43 & 3.58 \\
\hline$P$ for trend ${ }^{\mathrm{b}}$ & 0.031 & 0.274 & 0.100 & 0.002 \\
\hline \multicolumn{5}{|l|}{ Gender } \\
\hline Male & 3.64 & 3.33 & 3.44 & 3.58 \\
\hline Female & 3.65 & 3.32 & 3.39 & 3.54 \\
\hline$P^{\mathrm{a}}$ & 0.981 & 0.590 & 0.014 & 0.053 \\
\hline \multicolumn{5}{|l|}{ Race } \\
\hline White & 3.76 & 3.49 & 3.71 & 3.75 \\
\hline Black & 3.54 & 3.16 & 3.28 & 3.50 \\
\hline Hispanic & 3.63 & 3.37 & 2.99 & 3.22 \\
\hline$P^{\mathrm{a}}$ & $<0.001$ & $<0.001$ & $<0.001$ & $<0.001$ \\
\hline \multicolumn{5}{|l|}{ Education } \\
\hline$<$ High school diploma & 3.54 & 3.27 & 3.06 & 3.32 \\
\hline High school diploma & 3.57 & 3.28 & 3.36 & 3.52 \\
\hline Some college & 3.63 & 3.30 & 3.42 & 3.54 \\
\hline College graduate & 3.74 & 3.42 & 3.58 & 3.67 \\
\hline$P$ for trend ${ }^{\mathrm{b}}$ & $<0.001$ & $<0.001$ & $<0.001$ & $<0.001$ \\
\hline \multicolumn{5}{|l|}{ Income } \\
\hline$<\$ 24,999$ & 3.55 & 3.24 & 3.17 & 3.40 \\
\hline$\$ 25,000-\$ 49,999$ & 3.65 & 3.31 & 3.36 & 3.52 \\
\hline$\$ 50,000-\$ 74,999$ & 3.65 & 3.34 & 3.50 & 3.61 \\
\hline$\geq \$ 75,000$ & 3.77 & 3.49 & 3.71 & 3.75 \\
\hline$P$ for trend ${ }^{\mathrm{b}}$ & $<0.001$ & $<0.001$ & $<0.001$ & $<0.001$ \\
\hline $\begin{array}{l}\text { Neighborhood scales estit } \\
\text { (higher scores indicate better } \\
\text { a } P \text { value for difference in } \\
\text { using ANOVA models. } \\
\text { b } P \text { for trend represents } P\end{array}$ & $\begin{array}{l}\text { d using crude } \mathrm{m} \\
\text { hborhood conditic } \\
\text { an neighborhood }\end{array}$ & $\begin{array}{l}\text {. All neighbor } \\
\text { tor across cate } \\
\text { d as ordinal va }\end{array}$ & $\begin{array}{l}\text { od measures rar } \\
\text { ies of sociodem } \\
\text { bles into ANOY }\end{array}$ & $\begin{array}{l}\text { from } 1 \text { to } 5 \\
\text { raphic factors } \\
\text { model. }\end{array}$ \\
\hline
\end{tabular}

sisted after adjustment for socioeconomic indicators but were attenuated after adjustment for race/ethnicity. The reduction in the probability of being hypertensive associated with an increase from the 10th to the 90th percentile of neighborhood characteristics (relative prevalences ranging from 0.65 to 0.89 after adjustment for socioeconomic indicators) is equivalent to the effect of a reduction in age from 4 to 14 years. Thus, although the associations may appear small they are not trivial. To our knowledge, this is the first study to investigate associations of specific features of neighborhood environments with hypertension. The neighborhood walking environment and availability of healthy foods may affect hyperten- sion through their effects on diet and physical activity. Additionally, poor neighborhood safety and social cohesion may affect hypertension through physical activity and/or psychosocial stress. Future work will need to investigate the impact of neighborhood dimensions on these mediating pathways.

Associations were generally stronger for empirical Bayes estimation methods than for crude means. Because models with different predictors are not nested, the statistical significance of these differences cannot be determined. We cannot suggest a universally "best" method because the utility of each approach may vary from context to context; however, in general, empirical Bayes methods are generally useful 
TABLE 4. Adjusted Relative Prevalence $(95 \% \mathrm{Cl}$ ) of Hypertension Associated With a Difference in Neighborhood Score Equivalent to the Difference Between the 90th and the 10th Percentile by 4 Methods

\begin{tabular}{|c|c|c|c|}
\hline & Model $1^{\text {a }}$ & Model $2^{\mathrm{a}}$ & Model $3^{\mathrm{a}}$ \\
\hline \multicolumn{4}{|l|}{ Walking environment } \\
\hline Crude & $0.80(0.72-0.89)$ & $0.85(0.76-0.95)$ & $0.95(0.84-1.07)$ \\
\hline Empirical Bayes & $0.77(0.66-0.91)$ & $0.81(0.71-0.93)$ & $0.94(0.81-1.09)$ \\
\hline Conditional empirical Bayes & $0.67(0.58-0.78)$ & $0.75(0.64-0.88)$ & $0.90(0.78-1.05)$ \\
\hline Spatial empirical Bayes & $0.69(0.59-0.81)$ & $0.79(0.67-0.93)$ & $0.92(0.77-1.11)$ \\
\hline \multicolumn{4}{|l|}{ Healthy foods } \\
\hline Crude & $0.83(0.75-0.93)$ & $0.89(0.80-0.99)$ & $1.02(0.93-1.13)$ \\
\hline Empirical Bayes & $0.71(0.61-0.83)$ & $0.80(0.67-0.94)$ & $0.99(0.85-1.14)$ \\
\hline Conditional empirical Bayes & $0.65(0.55-0.76)$ & $0.72(0.61-0.85)$ & $0.94(0.80-1.12)$ \\
\hline Spatial empirical Bayes & $0.59(0.51-0.69)$ & $0.65(0.55-0.76)$ & $0.88(0.76-1.02)$ \\
\hline \multicolumn{4}{|l|}{ Safety } \\
\hline Crude & $0.77(0.68-0.87)$ & $0.88(0.77-1.00)$ & $0.93(0.83-1.04)$ \\
\hline Empirical Bayes & $0.71(0.61-0.83)$ & $0.77(0.66-0.89)$ & $0.98(0.84-1.15)$ \\
\hline Conditional empirical Bayes & $0.67(0.58-0.77)$ & $0.74(0.63-0.86)$ & $0.95(0.82-1.10)$ \\
\hline Spatial empirical Bayes & $0.67(0.57-0.79)$ & $0.75(0.63-0.88)$ & $0.99(0.84-1.16)$ \\
\hline \multicolumn{4}{|l|}{ Social cohesion } \\
\hline Crude & $0.83(0.74-0.93)$ & $0.89(0.79-1.01)$ & $1.02(0.90-1.15)$ \\
\hline Empirical Bayes & $0.72(0.60-0.86)$ & $0.80(0.67-0.96)$ & $0.91(0.76-1.08)$ \\
\hline Conditional empirical Bayes & $0.62(0.52-0.74)$ & $0.69(0.57-0.83)$ & $0.96(0.78-1.18)$ \\
\hline Spatial empirical Bayes & $0.80(0.66-0.97)$ & $0.92(0.76-1.12)$ & $1.08(0.92-1.28)$ \\
\hline \multicolumn{4}{|l|}{ Combined } \\
\hline Crude & $0.79(0.71-0.87)$ & $0.86(0.77-0.96)$ & $0.99(0.89-1.10)$ \\
\hline Empirical Bayes & $0.81(0.75-0.87)$ & $0.87(0.78-0.97)$ & $0.97(0.88-1.07)$ \\
\hline Conditional empirical Bayes & $0.75(0.68-0.83)$ & $0.80(0.72-0.88)$ & $0.92(0.83-1.02)$ \\
\hline Spatial empirical Bayes & $0.76(0.67-0.85)$ & $0.80(0.71-0.90)$ & $0.98(0.87-1.09)$ \\
\hline \multicolumn{4}{|c|}{$\begin{array}{l}\text { Categories for all control variables are listed in Table } 1 \text {. } \\
\text { Neighborhood scales range from } 1 \text { to } 5 \text {; Mean and standard deviation for all scales (crude, empirical Bayes, conditional } \\
\text { empirical Bayes, and spatial empirical Bayes) are as follows: }\end{array}$} \\
\hline \multicolumn{4}{|c|}{$\begin{array}{l}\text { Walking environment: } 3.59(0.43), 3.68(0.31), 3.65(0.31) \text {, and } 3.41(0.34) \text {; Availability of healthy foods: } 3.30(0.61), 3.22 \\
(0.37), 3.18(0.37) \text {, and } 3.23(0.42) \text {. } \\
\text { Safety: } 3.19(0.67), 2.89(0.52), 2.87(0.55) \text {, and } 3.06(0.56) \text {; Social cohesion: } 3.36(0.51), 3.04(0.32), 3.01(0.35) \text {, and } 3.05 \\
(0.38) \text {. } \\
\text { a Model 1: adjusted for site, age, sex; Model 2: site, age, sex, education, income; Model 3: site, age, sex, education, income } \\
\text { race/ethnicity. }\end{array}$} \\
\hline
\end{tabular}

when data are sparse, as in our case. The advantages of using these methods over the crude means are likely to be greatest when (1) data are sparse (unconditional empirical Bayes); (2) data are sparse and ecologic data on predictors of the construct of interest is available (conditional empirical Bayes); and (3) when data are sparse and there is strong spatial patterning (spatial empirical Bayes). In our case, there was little difference across the 3 empirical Bayes methods due to the high reliabilities of our neighborhood estimates, which resulted in relatively little shrinkage. In addition, neighborhood SEP was only a modest predictor of certain neighborhood features (availability of healthy foods, walking environment) and there was only modest spatial patterning in the constructs of interest. Additional work is needed to verify improvements yielded by empirical Bayes estimates in other settings.

The effects of neighborhood characteristics and the spatial scale of these effects may differ across regions. We found no evidence of heterogeneity by site; however, these analyses were limited by sample size and the range of scales within sites. Hence, we prefer to show estimates for the full sample adjusted for site so that the associations are across the full range of characteristics observed. However, we recognize that this approach may hide site heterogeneity.

A limitation of our study is the use of census tracts as proxies for neighborhoods. The geographic sizes of census tracts vary according to population density (they are larger in the Forsyth site than in the other 2 sites). In addition, census tracts may not be the geographic areas most relevant to hypertension. Unfortunately, there is little theory or data on which to base hypotheses regarding the relevant spatial scale. Area misspecification could have resulted in the relatively weak within-neighborhood clustering of hypertension that we documented (median OR $=1.47$ ). Despite limitations of census tracts, we believe our analyses are informative be- 
cause features of census tracts are likely to be correlated with features of the more specific areas relevant to the process being studied.

The cross-sectional design of our study limits our ability to infer causal associations. Because hypertension is cumulative, a history of exposure to neighborhood conditions is likely to be more relevant than exposure at a given time point. In our study, $46 \%$ of participants resided in the same neighborhood for at least 20 years with no significant interactions between time lived in neighborhood and neighborhood conditions. The absence of effect modification by time lived in neighborhood could have resulted from individuals being exposed to similar conditions in previous neighborhoods. An additional concern is that individuals may spend limited time in their neighborhood. In the Multi-Ethnic Study of Atherosclerosis, individuals reported spending an average of $75 \%$ of their time in their neighborhood during an average week.

Another limitation is the potential for measurement error due to the subjective report of neighborhood conditions. We previously reported differences in the ways in which individuals respond to the scale items based on their personal characteristics. For example, minorities were less likely to report violence than whites within the same areas. ${ }^{23}$ Variability due to individual subjectivity is averaged out by aggregating individual responses within a neighborhood, as we have done. The use of empirical Bayes estimates also allows improved estimation for neighborhoods by borrowing strength across areas.

Residual confounding by personal characteristics is always a possibility. We attempted to control for the key confounders of education and income. Although neighborhood characteristics were associated with income and education, there was sufficient overlap in these distributions to allow meaningful adjustment for these variables. Due to history of racial residential segregation in the United States, African Americans and other minorities are often spatially constrained to neighborhoods that are disadvantaged on multiple dimensions. In our analyses, associations of neighborhood characteristics with hypertension virtually disappeared after controlling for race/ethnicity. This finding results from the tight association between race/ethnicity and place characteristics in the United States context generally and in this sample in particular.

Race/ethnicity may be a confounder of associations between neighborhood factors and hypertension. It is also possible that neighborhood characteristics contribute to race/ ethnic differences in hypertension, and that race/ethnicity is a proxy for unmeasured neighborhood-level factors that covary with the ones we are investigating. If this is the case, race/ethnicity-adjusted associations of neighborhood characteristics with hypertension may actually underestimate causal neighborhood effects. Because of this complex issue we report associations before and after adjustment for race/ ethnicity.

By investigating specific attributes such as neighborhood walkability, availability of health foods, safety, and social cohesion, our analyses point to some of the processes through which environments may affect the development of hypertension. Causal inference regarding the presence of neighborhood health effects will benefit from more rigorous observational studies (including improved measurement of neighborhood attributes and longitudinal analyses), as well as from the analysis of natural experiments (such as changes in diet before and after the addition of a local supermarket to a particular neighborhood). This work will also benefit from studies that explore the complicated interplay between race/ ethnicity and place. If confirmed, our findings suggest that efforts to prevent hypertension may benefit from attention to the physical and social neighborhood environment.

\section{ACKNOWLEDGMENTS}

We thank the other investigators, the staff, and the participants of the Multi-Ethnic Study of Atherosclerosis (MESA) for their valuable contributions. A full list of participating MESA investigators and institutions can be found at http://www.mesa-nhlbi.org.

M. S. Mujahid created the study aims, conducted all analyses, and drafted the paper. A. V. Diez Roux contributed to the conceptualization and interpretation of the study findings, and assisted in writing sections of the paper. J. D. Morenoff and T. E. Ragunathan assisted with the interpretation of the statistical analyses. The remaining authors all equally contributed to reviewing drafts of the paper.

\section{REFERENCES}

1. Sundquist K, Winkleby M, Ahlen H, et al. Neighborhood socioeconomic environment and incidence of coronary heart disease: a follow-up study of 25,319 women and men in Sweden. Am J Epidemiol. 2004;159:655-662.

2. Diez Roux AV, Merkin SS, Arnett D, et al. Neighborhood of residence and incidence of coronary heart disease. N Engl J Med. 2001;345:99-106.

3. Diez-Roux AV, Nieto FJ, Muntaner C, et al. Neighborhood environments and coronary heart disease: a multilevel analysis. Am J Epidemiol. 1997; 146:48-63.

4. Diez Roux AV, Borrell LN, Haan M, et al. Neighbourhood environments and mortality in an elderly cohort: results from the cardiovascular health study. J Epidemiol Community Health. 2004;58:917-923.

5. Borrell LN, Diez Roux AV, Rose K, et al. Neighbourhood characteristics and mortality in the Atherosclerosis Risk in Communities Study. Int J Epidemiol. 2004;33:398-407.

6. Smith G, Hart C, Watt G, et al. Individual social class, area-based deprivation, cardiovascular disease risk factors, and mortality: the Renfrew and Paisley Study. J Epidemiol Community Health. 1998;52:399-405.

7. Diez Roux AV. Residential environments and cardiovascular risk. $J$ Urban Health. 2003;80:569-589.

8. Cubbin C, Hadden WC, Winkleby MA. Neighborhood context and cardiovascular disease risk factors: the contribution of material deprivation. Ethn Dis. 2001;11:687-700.

9. Sundquist J, Malmstrom M, Johansson SE. Cardiovascular risk factors and the neighbourhood environment: a multilevel analysis. Int $J$ Epidemiol. 1999;28:841-845.

10. Diez-Roux AV, Nieto FJ, Caulfield L, et al. Neighbourhood differences in diet: the Atherosclerosis Risk in Communities (ARIC) Study. J Epidemiol Community Health. 1999;53:55-63. 
11. Macintyre S, Ellaway A, Cummins S. Place effects on health: how can we conceptualise, operationalise and measure them? Soc Sci Med. 2002;55:125-139.

12. Diez Roux AV, Chambless L, Merkin SS, et al. Socioeconomic disadvantage and change in blood pressure associated with aging. Circulation. 2002;106:703-710.

13. Wilson DK, Kliewer W, Plybon L, et al. Socioeconomic status and blood pressure reactivity in healthy black adolescents. Hypertension. 2000; 35(1 pt 2):496-500.

14. Harburg E, Erfurt JC, Chape C, et al. Socioecological stressor areas and black-white blood pressure: Detroit. J Chronic Dis. 1973;26: 595-611.

15. Oakes JM. The (mis)estimation of neighborhood effects: causal inference for a practicable social epidemiology. Soc Sci Med. 2004;58:19291952.

16. O'Campo P. Invited commentary: advancing theory and methods for multilevel models of residential neighborhoods and health. Am J Epidemiol. 2003;157:9-13.

17. Diez Roux AV. Estimating neighborhood health effects: the challenges of causal inference in a complex world. Soc Sci Med. 2004;58:1953-1960.

18. van Kamp I, van Loon J, Droomers M, et al. Residential environment and health: a review of methodological and conceptual issues. Rev Environ Health. 2004;19:381-401.

19. Moore LV, Diez Roux AV. Associations of neighborhood characteristics with the location and type of food stores. Am J Public Health. 2006;96: 325-331.

20. Troped PJ, Saunders RP, Pate RR, et al. Associations between selfreported and objective physical environmental factors and use of a community rail-trail. Prev Med. 2001;32:191-200.

21. Gauvin L, Richard L, Craig CL, et al. From walkability to active living potential: an "ecometric" validation study. Am J Prev Med. 2005;28(2 suppl 2):126-133.

22. Raudenbush SW, Sampson RJ. Ecometrics: toward a science of assessing ecological settings, with applications to the systematic social observation of neighborhoods. Sociologic Methodol. 1999;29:1-41.

23. Mujahid MS, Diez Roux AV, Morenoff JD, et al. Assessing the measurement properties of neighborhood scales: from psychometrics to ecometrics. Am J Epidemiol. 2007;165:858-867.

24. Pikora TJ, Bull FCL, Jamrozik K, et al. Developing a reliable audit instrument to measure the physical environment for physical activity. Am J Prev Med. 2002;23:187-194.

25. Echeverria SE, Diez-Roux AV, Link BG. Reliability of self-reported neighborhood characteristics. J Urban Health. 2004;81:682-701.
26. Sampson RJ, Raudenbush SW, Earls F. Neighborhoods and violent crime: a multilevel study of collective efficacy. Science. 1997;277: 918-924.

27. Bild DE, Bluemke DA, Burke GL, et al. Multi-ethnic study of atherosclerosis: objectives and design. Am J Epidemiol. 2002;156:871-881.

28. Chobanian AV, Bakris GL, Black HR, et al. Seventh report of the Joint National Committee on Prevention, Detection, Evaluation, and Treatment of High Blood Pressure. Hypertension. 2003;42:1206-1252.

29. Chang JJ, Rabinowitz D, Shea S. Sources of variability in blood pressure measurement using the Dinamap PRO 100 automated oscillometric device. Am J Epidemiol. 2003;158:1218-1226.

30. Giles-Corti B, Donovan RJ. Socioeconomic status differences in recreational physical activity levels and real and perceived access to a supportive physical environment. Prev Med. 2002;35:601-611.

31. Stahl T, Rutten A, Nutbeam D, et al. The importance of the social environment for physically active lifestyle-results from an international study. Soc Sci Med. 2001;52:1-10.

32. Ball K, Bauman A, Leslie E, et al. Perceived environmental aesthetics and convenience and company are associated with walking for exercise among Australian adults. Prev Med. 2001;33:434-440.

33. Booth ML, Owen N, Bauman A, et al. Social-cognitive and perceived environment influences associated with physical activity in older Australians. Prev Med. 2000;31:15-22.

34. King AC, Castro C, Wilcox S, et al. Personal and environmental factors associated with physical inactivity among different racial-ethnic groups of U.S. middle-aged and older-aged women. Health Psychol. 2000;19: 354-364.

35. Raudenbush SW, Bryk AS. Hierarchical Linear Models: Applications and Data Analysis Methods. 2nd ed. Newbury Park, CA: Sage Publications; 2002.

36. Anselin L. Rate Transformations. Stat Support Document. Ann Arbor: Terra Seer; 2002.

37. Bithell JF. A classification of disease mapping methods. Stat Med. 2000;19:2203-2215.

38. Marshall RJ. Mapping disease and mortality rates using empirical Bayes estimators. J R Stat Soc Ser C Appl Stat. 1991;40:283-294.

39. Merlo J, Chaix B, Ohlsson H, et al. A brief conceptual tutorial of multilevel analysis in social epidemiology: using measures of clustering in multilevel logistic regression to investigate contextual phenomena. J Epidemiol Community Health. 2006;60:290-297.

40. Larsen K, Merlo J. Appropriate assessment of neighborhood effects on individual health: integrating random and fixed effects in multilevel logistic regression. Am J Epidemiol. 2005;161:81-88. 